\title{
The Role of Financial Instruments in Integrated Catastrophic Flood Management
}

\author{
Tatiana Ermolieva \\ International Institute for Applied Systems Analysis, Austria \\ Yuri Ermoliev \\ International Institute for Applied Systems Analysis, Austria \\ Guenther Fischer \\ International Institute for Applied Systems Analysis, Austria \\ Istvan Galambos \\ VITUKI Consult, Hungary
}

The main goal of this paper is to develop a flood management model that takes into account the specifics of catastrophic risk management: highly mutually dependent losses, the lack of information, the need for long-term perspectives and explicit analyses of spatial and temporal heterogeneities of various agents such as individuals, governments, and insurers. We use modified data from a pilot region of the Upper Tisza river, Hungary, to illustrate the evaluation of a public multi-pillar flood loss-spreading program involving partial compensation to flood victims by the central government, the pooling of risks through a mandatory public catastrophe insurance on the basis of location-specific exposures, and the demand for a contingent ex-ante credit to reinsure the insurance's liabilities. GIS-based catastrophe models and stochastic optimization methods are used to guide policy analysis with respect to location-specific risk exposures. We use economically sound risk indicators leading to convex stochastic optimization problems strongly connected with nonconvex insolvency constraint, VaR and CVaR (JEL G22, G28, C61).

Keywords: flood risk, catastrophe modeling, insurance, stochastic optimization, insolvency, contingent credit, $\mathrm{CVaR}$.

\footnotetext{
${ }^{*}$ We thank our anonymous reviewers, Editor-in-Chief and colleagues for their valuable comments and advice.
}

(Multinational Finance Journal, 2003, vol. 7, no. 3 \& 4, pp. 207-230)

(C) Multinational Finance Society, a nonprofit corporation. All rights reserved. DOI: $10.17578 / 7-3 / 4-5$ 


\section{Introduction}

Losses from human-made and natural catastrophes are rapidly increasing. Within the last three decades the direct damages from natural disasters have increased nine-fold, Borch (1999). The main reason for this is the clustering of people and capital in hazard-prone areas as well as the creation of new hazard-prone areas, a phenomenon that may be aggravated by a lack of knowledge of the risks. It is estimated that within the next 50 years more than a third of the world's population will live in seismically and volcanically active zones; see National Research Council (1999). Analysis of insurance companies shows that because of economic growth in hazard-prone areas, damages due to natural catastrophes have grown at an average annual rate of 5 percent, see Froot (1997).

This alarming human-induced tendency calls for integrated approaches to risk management allowing, in particular, to demonstrate that investments in risk management is a welfare generating strategy. The importance of integrated approaches for management of financial risks is emphasized in Mayers and Smith (1983) and Yang (2000). In particular, Yang (2000) argues that due to skewness of financial risks the Value-at-Risk ( $\mathrm{VaR}$ ) has become a very popular measure of risk. Catastrophes represent new challenges. Arrow (1996) admits that rare catastrophic risks affecting large communities cannot be properly treated by standard economic models. They call for robust combinations of various ex-ante and ex-post risk management decisions including deliberate selection of catastrophic risks for pooling by using appropriate stochastic optimization models; e.g., Borch (1999), Ermolieva et al.([1997) and Thomas (1994). In other words, simple basic ideas of risk pooling (Borch [1962]) are transformed into challenging stochastic decision-making problems (Erolieva [1997]).

This paper reports on the development of an integrated catastrophic risk management model necessary for the Upper Tisza river basin, which has been carried out as part of a joint IIASA-Sweden-Hungary project on flood risk management in the Upper Tisza Basin. The main issue is to address the specifics of catastrophic risks: highly mutually dependent endogenous losses, the lack of historical location-specific observations (unknown risks), the need for long-term perspectives, robust strategies, and explicit treatment of spatial and temporal heterogeneities of various agents such as individuals, governments and 
insurers. We propose a method allowing to deal with significant computational complexities of arising optimization problems.

The importance of integrated approaches is especially evident in Hungary, where 23 percent of the country is endangered by riverine floods. Only the Netherlands has a similar degree of risk, with 20 percent of the country under sea level. In Hungary and throughout the world, the losses from floods and other natural disasters are mainly absorbed by the immediate victims and their governments, e.g., Kleindorfer and Linnerooth-Bayer (2000) and Linnerooth-Bayer and Armendola (2000). The insurance industry and its premium payers also absorb a portion of catastrophic losses, but even in the wealthy countries this share is relatively small.

With increasing losses from floods, the Hungarian government is concerned with escalating costs for flood prevention, flood response, compensation to victims, and public infrastructure repair. Many government officials would like to increase the responsibility of individuals and local governments for flood risks and losses. Local governments may be more effective in the evaluation and enforcement of loss-reduction and loss-spreading measures, but this is possible only through location-specific ex-ante analysis of potential losses, the mutual interdependencies of these losses, and the sensitivities of the losses to new risk management strategies.

The lack of ex-ante designed strategies for dealing with increasing catastrophic losses was emphasized by Froot (1997) who concludes that losses from disasters

"....are paid ex-post by some combination of insurers and reinsurers (and their investors), insured, state and federal agencies and taxpayers, with only some of these payments being explicitly arranged ex-ante. This introduces considerable uncertainty about burden sharing into the system, with no particular presumption that the outcome will be fair. The result is incentives for players to shift burdens towards others, from the homeowner who builds on exposed coastline, to insurers who write risks that appear highly profitable in the absence of a large event ... But most importantly, bad or inefficient risk sharing raises the cost of capital for companies and requires returns for households, reducing the amount of profitable investments and the rate of growth of the economy.... it is worth noting that the gains from higher growth rate are huge ...."

For Hungary, facing special problems of a poor and immobile population, ex-ante mechanisms to fund the costs of recovery and, in 
particular, the establishment of a multi-pillar flood loss-sharing program, are especially important.

In this paper we illustrate the design of such a program assuming that for the first pillar the government would provide compensation of a limited amount to all households that suffer losses from flooding. As a second pillar, a mutual catastrophe fund would be established by pooling risks through a mandatory public catastrophe flood insurance on the basis of location-specific risk exposures. It is assumed that the governmental financial aid is regulated through this fund. As a third pillar, a contingent credit may also be available to provide an additional injection of capital to stabilize the system.

The analysis of possible gains and losses from different arrangements of the program is a multi-disciplinary task, which takes into account the frequency an intensity of location-specific hazards, the stock of capital at risk, its structural characteristics, feasible decisions and different measures (in particular, engineering, financial) of vulnerability. These efforts require the development of the so-called catastrophe models, seeWalker (1997).

The aim of catastrophe models is to generate potential samples of mutually dependent losses for a given vector of policy variables. For example, when there is a lack of historical data, models can estimate distributions of losses and gains for different locations, households, insurers, and governments. This is critically important in the case of rare events or new policies that have never been implemented in practice. The catastrophe model opens up the possibility for "if - then" analysis, which allows the evaluation of a finite number of policy alternatives. However, these analyses may run quickly into an infinite number of possible combinations (see discussion in Section 3).

As Walker (1997) admits, there is an urgent need for incorporating catastrophe models into more comprehensive catastrophic risk management models. The main goal of this paper is to demonstrate such a possibility by using data from the case study in the Upper Tisza basin. We slightly modified initial data in order to better illustrate implications for financial ex-ante decisions. In particular, we selected a specific loss-sharing program among many others analyzed for this region. For an analysis of other loss-sharing programs for seismic prone regions in Italy and Russia see Amendola et al. (2000 a,b) and Ermoliev et al. (2001).

Section II discusses the main features of a GIS-based catastrophe model developed for the Upper Tisza pilot region that simulates samples 
of potential location-specific losses. Traditional insurance and finance quantify extreme events in monetary units Embrechts et al. (2000). The proposed catastrophe model deals with non-quantifiable (in this sense) events and multivariate distributions of extreme values, i.e., with the cases which are not sufficiently treated within the conventional extreme value theory.

Section III discusses the shortcomings of "if-then" analysis and outlines general ideas of Adaptive Monte Carlo (AMC) optimization proposed by Ermolieva (1997) and Ermolieva et al. (2000). The AMC optimization technique is compared with known AMC simulation proposed by Pugh (1966) to improve only the efficiency of the sampling. The AMC optimization is used for solving analytically intractable optimization problems which require decision-specific simulations for estimating of objective and constraints functions. The use of other search methods, such as genetic algorithms is practically impossible due to time-consuming evaluation of intermediate solutions.

Section IV describes a spatial and dynamic stochastic optimization model for evaluation of the selected public multipillar flood loss spreading program in the Upper Tisza region. The model emphasizes the cooperation of various agents in dealing with catastrophes. The solution to catastrophic risk management, especially in small economies with limited risk absorption capacity, cannot be accomplished without the pooling of risk exposures; see the discussion in Pollner (2000) and Cummins and Doherty (1996). The model involves pooling risks through mandatory public catastrophe flood insurance based on location-specific exposures, partial compensation to the flood victims by the central government, and a contingent credit to the mandatory public insurance. Definitely this program encourages accumulation of own regional capital to better "buffer" against the involved risks. In order to stabilize the program we use economically sound risk indicators such as expected overpayments by "individuals" (cells of flood-prone areas) and an expected shortfall of the mandatory insurance. We use these indicators together with the so-called stopping times to orient the analysis towards the most destructive scenarios. The explicit introduction of ex-post borrowing as a measure against insolvency enables us to approximate the insolvency constraint by a convex optimization problem, whereas the use of the contingent credit leads to the Conditional-Value-at-Risk (CVaR) type of risk measures.

Numerical experiments in section $\mathrm{V}$ indicate a strong dependence of demand for contingent credit on the composition of other risk 
management decisions.

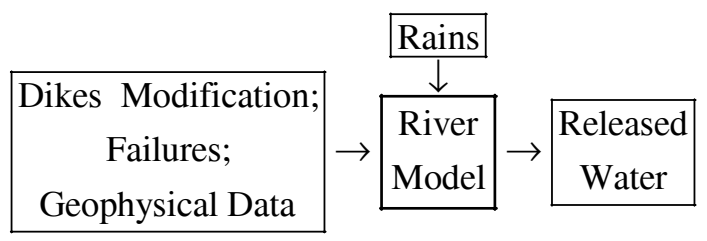

River Module

\begin{tabular}{|c|} 
Geo-Physical \\
Spatial Data; \\
Released Water
\end{tabular}$\rightarrow \begin{gathered}\text { Spatial } \\
\text { Induction } \\
\text { Model }\end{gathered} \rightarrow \begin{gathered}\text { Standing } \\
\text { Waters }\end{gathered}$

Spatial Inundation Module

\begin{tabular}{|c|}
$\begin{array}{c}\text { Standing Waters; } \\
\text { Feasible Decisions; } \\
\text { Economic Data }\end{array} \rightarrow \begin{array}{c}\text { Vulnerability } \\
\text { Models }\end{array} \rightarrow \begin{array}{l}\text { Direct } \\
\text { Spatial } \\
\text { Losses }\end{array}$ \\
\hline
\end{tabular}

Vulnerability Module

\begin{tabular}{l}
$\begin{array}{l}\text { Feasible Decisions; } \\
\text { Direct Spatial Losses }\end{array} \rightarrow$ MAAS $\rightarrow \begin{array}{c}\text { Losses and Gaines } \\
\text { of Agents }\end{array}$ \\
\hline
\end{tabular}

Multi-Agent Accounting System (MASS)

\begin{tabular}{|c|}
$\begin{array}{c}\text { Feasible } \\
\text { Decisions }\end{array}$ \\
Model
\end{tabular}$\rightarrow \begin{gathered}\text { Monte-Carlo } \\
\text { Model } \\
\text { of Losses and Gaines }\end{gathered}$

Variability Module

FIGURE 1.-Modules of Catastrophe Model

\section{Catastrophe Model}

As is shown in figure 1, the catastrophe model developed for the pilot region of the Upper Tisza river consists of five sub-models (modules): the "River" module, the "Inundation" module, the "Vulnerability" module, the "Multi-Agent Accounting System", and the "Variability" module. 
The river module calculates the volume of discharged water to the pilot region from different river sections for given heights of dikes, given scenarios of their failures or removals, and rainfalls. The latter are modeled by upstream discharge curves. Thus, formally, the River module maps an upstream discharge curve into the volume of water released to the region from various sections. The underlying sub-model is able to estimate the discharged volume of the water into the region under different conditions, for example, if the rain patterns change, if the dikes are heightened, or if they are strengthened or removed.

The next module is the spatial GIS-based Inundation sub-model. For the pilot region it contains 1500 by 1500 grids. This module maps water released from the river into levels of standing water and thus it can estimate the area of the region affected by different decisions.

The vulnerability module maps spatial patterns of released water into economic losses. This module calculates direct losses and may include possible cascading effects, such as floods causing fire and its consequences. It may also include loss reduction measures, e.g., new land-use modifications and flood preparedness measures. This module is able to indicate changes in economic losses from changes in risk reduction measures.

The multi-agent accounting system (MASS) module maps spatial economic losses into gains and losses of agents. These agents are the central government, a mandatory catastrophe insurance (pool) and "individuals" (cells).

Given sufficient data, the aforementioned sub-models can generate scenarios of losses and gains at different locations for specific scenarios of failures, rainfalls, risk reduction measures and risk spreading schemes. But there are significant uncertainties and a considerable variability in these losses and gains. For example, a 50-year flood may occur in 5 days or in 70 years.

Insurers are particularly concerned about variability since they may not have the capacity to cover large losses. In an attempt to maintain their solvency, they may charge higher premiums, which may result in overpayments by the insured. Alternatively, insurers may undercharge contracts.

Insurers are also concerned about loss-reduction measures. A higher dike may fail and cause more damages in comparison to a dike without modification. The Variability module, a Monte Carlo model, transforms spatial scenarios of losses and gains among agents into histograms of probability distributions. For example, it derives histograms of direct 
losses at a location or a subregion. It also calculates histograms of overpayments and underpayments for different agents (see section V).

\section{Adaptive Monte Carlo Optimization}

The following simple example illustrates shortcomings of the straightforward "if-then" scenario analysis. Let us assume that an

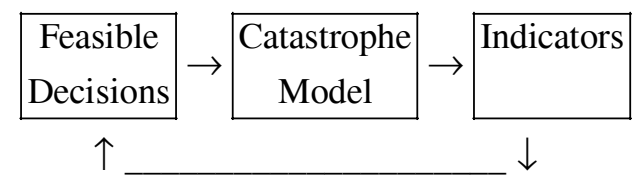

FIGURE 2. The Adaptive Monte Carlo Optimization Model

insurer in the region can have different policies regarding the extent of coverage that it offers, say $0 \%, 10 \%, 20 \%, \ldots, 100 \%$, i.e., altogether eleven alternatives. For ten locations the number of possible policy scenarios is $10^{11}$. With one second per module run, the computer time required for the evaluation approaches 100 years. Therefore, with 100 locations the straightforward "if - then" analysis runs into eternity. The same computational complexity arises in dealing with location-specific premiums or investments in different segments of dikes.

The fundamental question concerns the evaluation of a desirable policy without the exact evaluation of all the options. The complexity of this task is due to analytical intractability of stochastic catastrophe models, generating only random values of goal functions and often requiring a large number of simulations for estimating outcomes of a single decision.

The standard optimization methods imply that the goal (objective and constraints) functions are exactly calculated, i.e., for a given feasible solution these functions are calculated without an additional sampling procedure. Therefore, in general cases, we have to rely on the stochastic optimization methods in particular on the so-called adaptive Monte Carlo optimization, e.g., Ermolieva et al. (2003), Ermolieva (1997) and Ermoliev et al. (2000).

"Adaptive Monte Carlo" is a technique that makes on-line use of sampling information to sequentially improve the efficiency of the sampling itself, see Pugh (1966). We use "adaptive Monte Carlo optimization" in a rather broad sense, i.e. the efficiency of the sampling 
procedure is considered as a part of more general on-line improvements with respect to different decision and goals.

The adaptive Monte Carlo optimization model for the Upper Tisza region consists of three interacting blocks: feasible decisions, the Monte Carlo catastrophe model, and indicators; see figure 2.

The block feasible decisions represents all feasible policies for coping with floods. In general, they may include feasible heights of dikes, insurance coverage, land use modifications, etc. These variables affect performance indicators such as profits of insurers, underpayments or overpayments by the insured, costs, insolvency and stability indicators.

The essential feature is the feed-back mechanism updating decisions towards specific goals. The updating procedure relies on stochastic optimization techniques as is discussed in section V. Losses are simulated by the catastrophe model, causing an iterative revision of the decision variables after each simulation run. In a sense, the adaptive Monte Carlo optimization simulates in a remarkably simple and evolutionary manner the learning and adaptation process on the basis of the simulated history of catastrophic events.

\section{The Stochastic Optimization Model}

Stochastic optimization provides a framework for the iterative revision of decisions embedded in the catastrophe model. These decisions influence the contribution of location-specific risks on the overall catastrophe losses. In the model for the Upper Tisza region we use approaches similar to those in Amendola et al. (2000), Emolieva et al. (1997) and Ermoliev et al. (2000, 2001).

The main idea is based on subdividing the study region into $m$ cells, $j=1,2, \ldots, m$. These cells may correspond to a collection of households at a certain site, a collection of grids (zones) with a similar land-use structure, or an administrative district or a grid with a segment of a gas pipeline.

The choice of cells provides a desirable representation of losses. In our case, the cells consist of the value of the physical structures. Catastrophes, which are simulated by the catastrophe model, affect at random different cells and produce mutually dependent at time $t$ losses $L_{j}^{t}$. These losses can be modified by various decision variables. Some of the decisions reduce losses, say a dike, whereas others spread them 
on a regional, national, and international level, e.g., insurance contracts, catastrophe securities, credits, and financial aid.

If $x=\left(x_{1}, x_{2}, \ldots, x_{n}\right)$ is the vector of the decision variables, then $L_{j}^{t}$ fro cell $j$ and time $t$ depend on the decision variables $x$, i.e., $L_{j}^{t}(x)$. In the case of the Tisza river, for example, we can think of $L_{j}^{t}(x)$ as $L_{j}^{t}$ being affected by the decisions of the insurance to cover location-specific fractions (or layers) of losses $L_{j}^{t}, j=1,2, \ldots, m$.

In the most general case, vector $x$ comprises decision variables of different agents, including governmental decisions, such as the height of a new dike or a public compensation scheme defined by a fraction of total losses $\Sigma_{j} L_{j}^{t}$. The insurance decisions concern premiums paid by individuals and the payments of claims in the case of catastrophe. There are complex interdependencies among these decisions, which call for the cooperation of agents.

For example, the partial compensation of catastrophe losses by the government enforces decisions on loss reductions by individuals and, hence, increases the insurability of risks, and helps the insurance to avoid insolvency. On the other hand, the insurance combined with individual and governmental risk-reduction measures can reduce losses, compensations and government debt and stabilize the economic growth of the region and the wealth of individuals.

In the following we do not consider the most general situation, e.g., we consider only the proportional compensation by the government, proportional insurance coverages, and we do not use discount factors. The long time horizons of our model require new approaches to the discounting, since traditional discount factors (typical for the existing financial markets) dramatically reduce values beyond a few decades. In fact, the use of so-called stopping time in our model induces implicit discounting associated with the time spans of catastrophic events, see Ermoliev and Wets (1988). Unfortunately, the discussion of this important fact is beyond the scope of this paper.

In this application the system is modeled until a first catastrophic flood, which occurs within a given time horizon. We define this moment as the stopping time. For the Upper Tisza region this event is associated with the break of a dike that may occur only after a 100-year, 150- or 1000 -year flood. They are characterized by so-called upstream discharge curves and the probability of breaking each of the three dikes. The discharge curves characterize the amount of water incoming in time to the given section of the river. The timing of the first catastrophic flood significantly affects the accumulation of risk reserves by the insurance, 
and total payments of individuals; for example, a 100-year flood with the break of a dike may occur in two years.

Let $\tau$ be a random (stopping) time to a first catastrophe within a time interval $[0, T]$, where $T$ is some planning horizon, say 50 years. If no catastrophe occurs, then $\tau=T$. Since $\tau$ is associated with the break of a dike, the probability distribution of $\tau$ is, in general, affected by some components of vector $x$, e.g., by decisions on dike modifications, the land use changes, building reservoirs, etc. In this paper we discuss only the case when $\tau$ does not depend on $x$, i.e., we assume that the distribution of $\tau$ follows a mixed geometric distribution according to identified scenarios of floods and probabilities of dike breaks.

Let $L_{j}^{\tau}$ be random losses at location $j$ at time $t=\tau$. In our analysis we evaluate the capacity of the mandatory public catastrophe insurance in the upper Tisza region only with respect to financial loss-spreading decisions. Let us use a special notation for their components such as $\pi_{j}$, $\varphi_{j}, v$ and $y$. If $\pi_{j}$ is the premium rate paid by location $j$ to the mandatory insurance, then the accumulated mutual catastrophe fund at time $\tau$ together with the proportional compensation $v \Sigma_{j} L_{j}^{\tau}$ by the government is equal to

$$
e_{1}=\tau \sum_{j} \pi_{j}+v \sum_{j} L_{j}^{\tau}-\sum_{j} \varphi_{j} L_{j}^{\tau}
$$

where $0 \leq \varphi_{j} \leq 1$, is the percent of insurance coverage for cell $j$. Thus, the pair $\left(\pi_{j}, \varphi_{j}\right)$ defines the insurance contract with a location $j$. We assume that the compensation to victims by the government is paid through the mandatory public insurance. The stability of the insurance program depends on whether the accumulated mutual fund together with the governmental compensation is able to cover claims, i.e., on the probability of event $e_{1} \geq 0$.

The stability also depends on the willingness of individuals to accept premiums, i.e., with the probability of overpayments:

$$
e_{2}=\tau \pi_{j}-\varphi_{j} L_{j}^{\tau} \geq 0
$$

for $j=1,2, \ldots, m$. Apart from the compensation $v \Sigma_{j} L_{j}^{\tau}(x)$, the government arranges a contingent credit $y$ with a fee $q$ to improve the stability of the mandatory insurance by transforming event (1) into (3):

$$
e_{3}=\tau \sum_{j} \pi_{j}+v \sum_{j} L_{j}^{\tau}-\sum_{j} \varphi_{j} L_{j}^{\tau}+y-\tau q y \geq 0
$$


Here we assume that the mandatory insurance pays the fee $\tau \pi q$ as long as there is no occurrence of dike break. If the break occurs, the mandatory insurance rapidly receives the credit $y$, whereas the government pays back $y$. The advantages of this financial arrangement in contrast to catastrophic bonds are discussed in Pollner (2000).

The difference between compensation $v \Sigma_{j} L_{j}^{\tau}$ and contingent credit $y$ is significant: the outflow of fees is smooth, whereas the compensation of claims has a sudden impact at time $\tau$, and without $y$ it may require a higher government compensation (greater $v$ ) possibly exceeding the available budget. Therefore, without ex-ante contingent injections of capital $y$ the diversion of capital from other governmental needs may occur.

Let us note that the budget constraint, which is not considered explicitly in this model, raises a general question on the optimal dynamic management of the available budget in order to increase the stability of the mandatory insurance and its efficiency. For example, besides the contingent credit, a reasonable option may also be to invest some money in liquid assets. The main aim of our analysis in this paper is narrower: the evaluation of the mandatory public insurance capacity and the demand for contingent credit.

Inequalities (2)-(3) define important events, which constrain the choice of the decision variables specifying the insurance program, i.e., the compensation rate $v$ by the government, coverages by the insurance company $\varphi_{j}$, premiums $\pi_{j}$, and credit $y$ with fee $q$. Let us emphasize that in the contract $\left(\pi_{j}, \varphi_{j}\right)$ the premium $\pi_{j}$ is treated as a decision variable satisfying "fair" equilibrium equations (2)-(4). As we show in Section 5 the use of standard actuarial premiums may not be appropriate for catastrophic risks. The values of $e_{2}$ and $e_{3}$ and likelihood of events (2)-(3) determine the stability (resilience) of the program. In a rough way this can be expressed in terms of the probabilistic constraint

$$
P\left(e_{2} \geq 0, e_{3} \leq 0\right) \leq p
$$

where $p$ is a desirable probability of the program's default, say a default that occurs only once in 100 years. Constraint (4) is similar to the so-called insolvency constraint, a standard for regulations of the insurance business, see Stone (1973). In the stochastic optimization constraint (4) is known as the chance constraint, see Ermolieva et al. (2003). Unfortunately, this constraint does not account for the values $e_{2}$ and $e_{3}$ which is important for the government, since it cannot ignore 
the region in distress.

Let us assume that vector $x$ consists of the components $\pi_{j}, \varphi_{j}$ and $y$. The main goal is formulated as the minimization of expected total losses

$$
F(x)=E \sum_{j}\left(1-\varphi_{j}\right) L_{j}^{\tau}+\gamma\left(v E \sum_{j} L_{j}^{\tau}+y\right)
$$

subject to the chance constraint (4).

The expected losses function $F(x)$ requires further discussion. It reflects the government's interest to reduce uncovered losses and, at the same time, to minimize the governmental payments $v E \Sigma_{j} L_{j}{ }^{\tau}+y$ with a weight $0 \leq \gamma \leq 1$. In fact, the evaluation of optimal $v$ and $y$ requires explicit introduction of the governmental catastrophe budget and its insolvency constraint similar to (2)-(4).

The size of this budget is itself a key decision variable, which calls for essential modifications of the model. Therefore, in our numerical experiments in section $\mathrm{V}$, we use a practically feasible option for $v$ and analyze only the demand for contingent credit $y$. This is derived from the evaluation of mandatory public catastrophe insurance to sustain against catastrophic floods.

Constraint (4) imposes significant methodological challenges even in cases when $\tau(x)$ does not depend on $x$ and events (2)-(3) are defined by linear functions of decision variables; see discussion in Ermoliev and Wets (1988), p. 8, and Ermoliev et al. (2000, 2003). This constraint is of "black-and-white" character, i.e., it accounts only for a violation of (2)-(3) and not for its size.

There are important connections between the minimization of $F(x)$ subject to highly non-linear and possibly discontinuous chance constraints (4) and the minimization of convex functions, which have important economic interpretation. Consider the following function

$$
\begin{aligned}
G(x)=F(x)+a E & \max \left\{0, \sum_{j} \varphi_{j} L_{j}^{\tau}-v \sum_{j} L_{j}^{\tau}-\tau \sum_{j} \pi_{j}-y+\tau q y\right\} \\
& +\beta E \sum_{j} \max \left\{0, \tau \pi_{j}-\varphi_{j} L_{j}^{\tau}\right\}
\end{aligned}
$$

where $\alpha$ and $\beta$ are positive parameters. This function is affected in a rather complex manner by probability distributions of $L_{j}^{\tau}$ and $\tau$ modified by decisions $\pi_{j}, \varphi_{j}, v$ and $y$.

It is possible to show that for large enough values of $\alpha$ and $\beta$, the 
minimization of function $G(x)$ generates solutions $x$ with $F(x)$ approaching the minimum of $F(x)$ subject to (4) for any given level $p$; see Ermolieva et al. (2003), ch. 2.

The minimization of $G(x)$ defined by (5) has a simple economic interpretation. Function $F(x)$ comprises expected direct losses associated with the insurance program. The second term includes the expected shortfall of the program to fulfill the obligations; it can be viewed as the expected amount needed for this purpose of ex-post borrowing with a fee $\alpha$. Similarly, the third term can be interpreted as the expected ex-post borrowing with a fee $\beta$ needed to compensate overpayments. Obviously that large enough fees $\alpha$ and $\beta$ will tend to preclude the violation of (2)-(3). Thus, the ex-post borrowing with large enough fees allows for a control of the insolvency constraints (4).

It is easy to see that the use of the ex-post borrowing (expected shortfall) in the second term of $G(x)$ in combination with the optimal ex-ante contingent credit $y$ controls the $\mathrm{CVaR}$ type risk measures. Indeed, the minimization of $G(x)$ is an example of stochastic min-max problems, see Ermolieva et al. (2003), ch. 22.

By using standard optimality conditions for these problems we can derive the optimality conditions for the contingent credit . For example, assuming continuous differentiability of $G(x)$ which follows in particular from the continuity of underlying probability distributions of $L_{j}^{t}$, despite non-smooth random functions under the expectation $E$, it is easy to see that the optimal level of the credit $\gamma>0$ satisfies the equation

$$
\frac{\partial G}{\partial \gamma}=\gamma-\alpha P\left(\sum_{j} \varphi_{j} L_{j}^{\tau}-v \sum_{j} L_{j}^{\tau}-\tau \sum_{j} \pi_{j}>y\right)=0
$$

Thus, the optimal amount of the contingent credit is defined as a quantile of the random variable $\Sigma_{j} \varphi_{j} L_{j}^{\tau}-v \Sigma_{j} L_{j}^{\tau}-\tau \Sigma \pi_{j}$ specified by the ratio $\gamma / \alpha$, which has to be not greater than one. Hence, the expectation in the second term of $G(x)$ for optimal $y$ is taken under the condition that $y$ is the quantile of $\Sigma_{j} \varphi_{j} L_{j}^{\tau}-v \Sigma_{j} L_{j}^{\tau}-\tau \Sigma \pi_{j}$. This is in accordance with the definition of CvaR, see Artzner et al. (1999) and Rockafellar and Uryasev (2000). More general risk measures emerge from the optimality conditions of $G(x)$ with respect to premiums $\pi_{j}$ and $\varphi_{j}$.

The importance of such an economically sound risk measure as expected shortfall was emphasized by many authors, e.g., Artzner et al. (1999), Embreechts et al. (2000), Jobst and Zenios (2001), and Rockafellar and Uryasev (2000). Important connections of CVaR with 
the linear programs were discussed in Artzner et al. (1999) and Rockafellar and Uryasev (2000).

Let us note that $G(x)$ is a convex function in the case when $\tau$ and $L_{j}^{\tau}$ do not depend on $x$. In this case the stochastic minimax problem (5) can be approximately solved by linear programming methods; see the general discussion in Ermolieva et al. (1997). The main challenge is concerned with the case when $\tau$ and $L_{j}^{\tau}$ are implicit functions of $x$. Then we can only use the adaptive Monte Carlo optimization. Let us outline only the main idea of these techniques. More details and further references can be found in Ermolieva (1997), Ermoliev et al. (2000 and 2001), and Ermoliev and Wets (1988).

Assume that vector $x$ incorporates not only risk management decision variables but also includes components affecting the efficiency of the sampling itself. An adaptive Monte Carlo procedure searching for a solution minimizing $G(x)$ of type (5) starts at any reasonable guess $x^{0}$. It updates the solution sequentially at steps $k=1,2, \ldots$ by the rule $x^{k+1}$ $=x^{k}-\rho_{k} \xi^{k}$, where numbers $\rho_{k}$ are step-sizes satisfying the condition

$$
\sum_{k=0}^{\infty} \rho_{k}=\infty \text { and } \sum_{k=0}^{\infty} \rho_{k}^{2}=\infty
$$

For example, the specification $\rho_{k}=(1 / k)+1$ would fit.

The random vector $\xi^{k}$ is an estimate of the gradient $G_{x}(x)$ or its analogs for nonsmooth function $G(x)$. This vector is easily computed from random observations of $G(x)$. For example, let $G^{k}$ be a random observation of $G(x)$ at $x=x^{k}$ and $\tilde{G}^{k}$ be a random observation of $G(x)$ at $x=x^{k}+\delta_{k} h^{k}$. The numbers $\delta_{k}$ are positive, $\delta_{k} \rightarrow 0, k \rightarrow \infty$, and $h^{k}$ is an independent observation of the vector $h$ with independent and uniformly distributed on $[-1,1]$ components. Then $\xi^{k}$ can be chosen as

$$
\xi^{k}=\left[\left(\tilde{G}^{k}-G^{k}\right) / \delta^{k}\right] h^{k}
$$

The formal analysis of this method, in particular, for discontinuous goal functions, is based on general ideas of the stochastic quasigradient methods; see Ermolieva et al. (2003) for further details.

\section{Demand for Ex-ante Contingent Credit}

According to the model of section IV, the demand of the pilot region for 
a contingent credit significantly depends on the capacity of the mandatory public insurance to sustain against catastrophic floods, the fraction $v$ of the government compensation and on the interplay of various factors, e.g., on the occurrence of floods and the reliability of the flood protection system (e.g., dike failures). In this section we discuss some numerical experiments using a modification of real data collected in the Upper Tisza region. The main purpose of the following discussion is not the results of our findings, but rather the illustration of the proposed model.

The case study region consists of $1,500 \times 1,500$ grids, and for each grid there are data on the vulnerability of its content. These grids are further aggregated into 40 cells.

The flood occurrences are modeled according to specified scenarios of catastrophic floods and dike breaks. There are three dikes allocated along the pilot river branch. Each of them may break under the probability of a 100-year, 150-year, and 1,000-year flood.

In this paper, we take into account only structural losses. The simulation time horizon is assumed to be 50 years. The number of simulations (scenarios) in a single experiment was 10,000. A contingent credit in our model is introduced to stabilize equation (1) according to equation (3). The demand for the credit is, therefore, defined by negative values of indicator $e_{1}$ or $e_{3}$ for optimal (or given) solutions $\varphi_{j}, \pi_{j}$ minimizing (5) for $y=0$ and given $v$. This defines also the lack of capacity for the mandatory insurance.

Figures 3 and 4 illustrate the results of the experiments with $v=0.25$ and $y=0$. The horizontal axis shows the total demand for contingent credit, negative $e_{1}$, whereas the vertical axis shows the number of simulations and the cumulative probability.

In the numerical experiments we analyzed the capacity of mandatory insurance and the demand for the credit under different assumptions on premiums $\pi_{j}, j=1,2, \ldots, m$, where $m$ is the number of cells. In our case $m=40$.

1. Premiums calculated according to the actuarial principle based on location-specific average losses. Coverages $\varphi_{j}$ are derived from the minimization of function (5).

2. "Fair" premiums are calculated by minimizing function (5) with respect to $\pi_{j}$ and $\varphi_{j}$. This accounts for the distribution of losses for each location and the "equilibrium" defined by equations (2) and (3). 


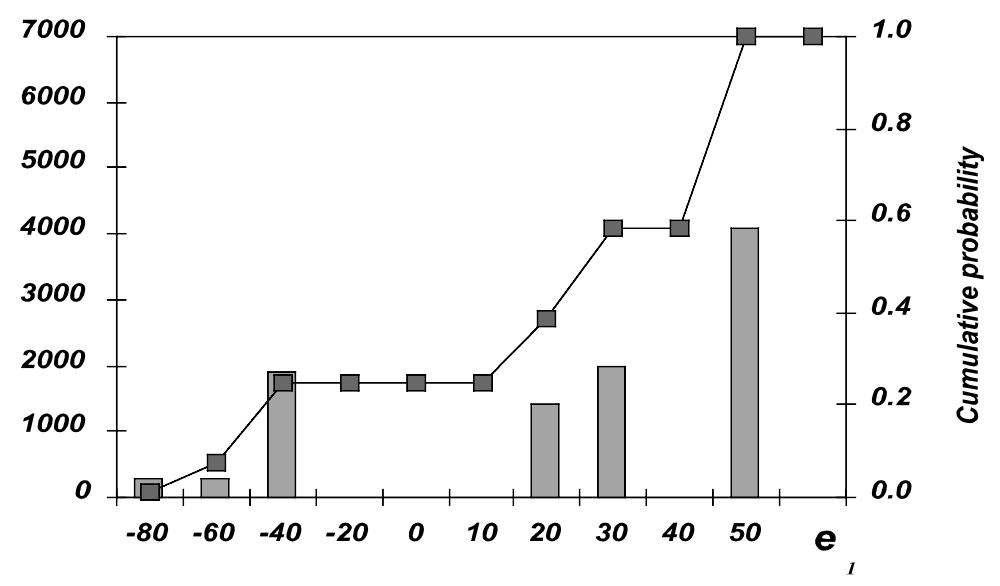

FIGURE 3.-Actuarial Premiums.

In practical calculations histograms for constraints (4) calculated simultaneously with the minimization of (5) provide a signal for increasing or decreasing "penalties" (risk factors) $\alpha$ and $\beta$ to achieve a satisfactory level $p$. Intuitively, greater $\alpha$ and $\beta$ values lead to constraints (4) with smaller $p$. On the other hand, this may considerably reduce insurance coverages of catastrophic exposures. A trade-off between these two effects can be resolved by using some additional considerations, e.g., political considerations or purely visual character of histograms, which cannot be formalized in general within a single model. This is similar to the standard welfare analysis.

According to our experiments, the premium for the first option equals on average (per location and year) 0.87 million HF (Hungarian forints) (exchange rate: one HF equals 0.003302 U.S. dollars). Coverages are derived by minimization (5) for $\alpha=\beta=N=10$. As we can see from figure 3 , the inflow of premiums is not enough to compensate the losses, since $e_{1}$ is often negative, which defines a certain safety (solvency) level $p$ for constraint (5). It is clear that in more than 2,000 scenarios out of 10,000 of simulated catastrophic events the mandatory insurance lacks the capacity to cover losses. This calls for a more significant intervention by the government through either increasing level compensations $v$, and/or through the contingent credit.

"Fair" premiums improve the situation. Figure 4 illustrates the changes in the total demand for contingent credit by using the optimal 


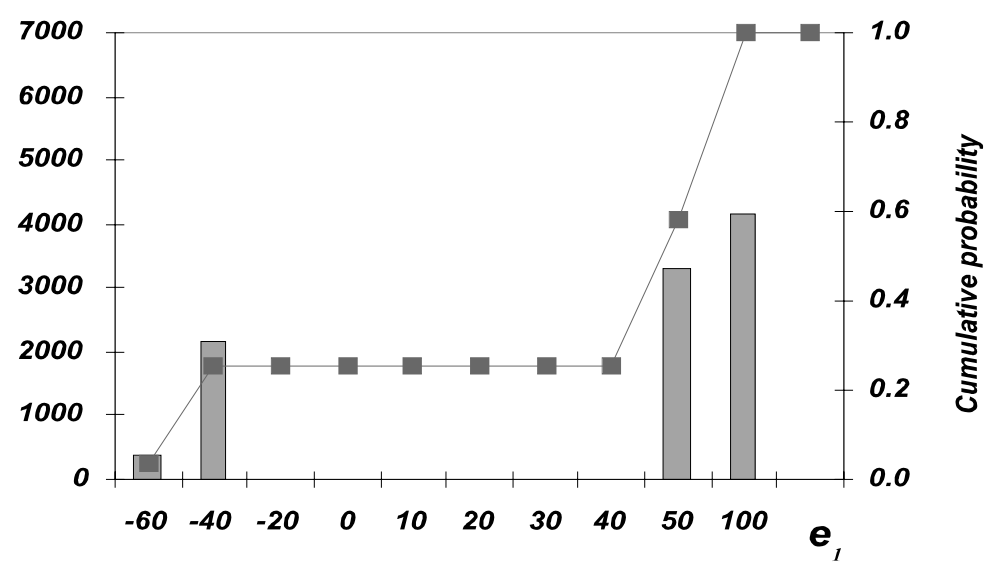

FIGURE 4.-Optimal Premiums.

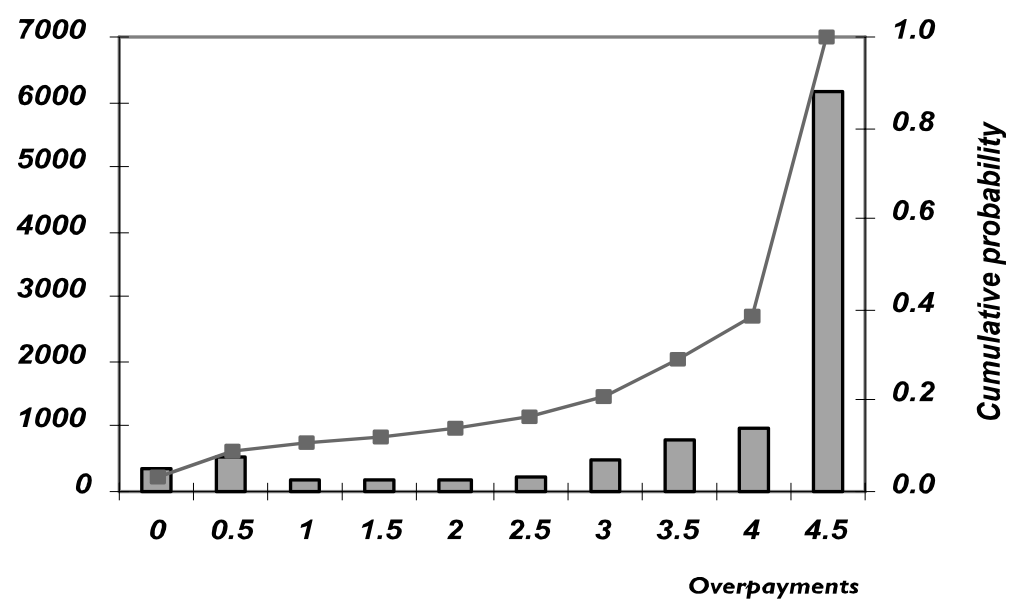

FIGURE 5.-Actuarial Premiums.

premiums calculated from the minimization of (6) for the same $v=0.25$, $y=0$ and $N=10$. The model suggests a premium rate on average (per location and year) equal to 0.83 million HF, which is lower than in the first case.

Figure 4 shows that the demand for contingent credit is also reduced (fewer negative values on the horizontal axis). "Fair" premiums improve also overpayments per year (see figures 5 and 6), i.e., the distribution of 


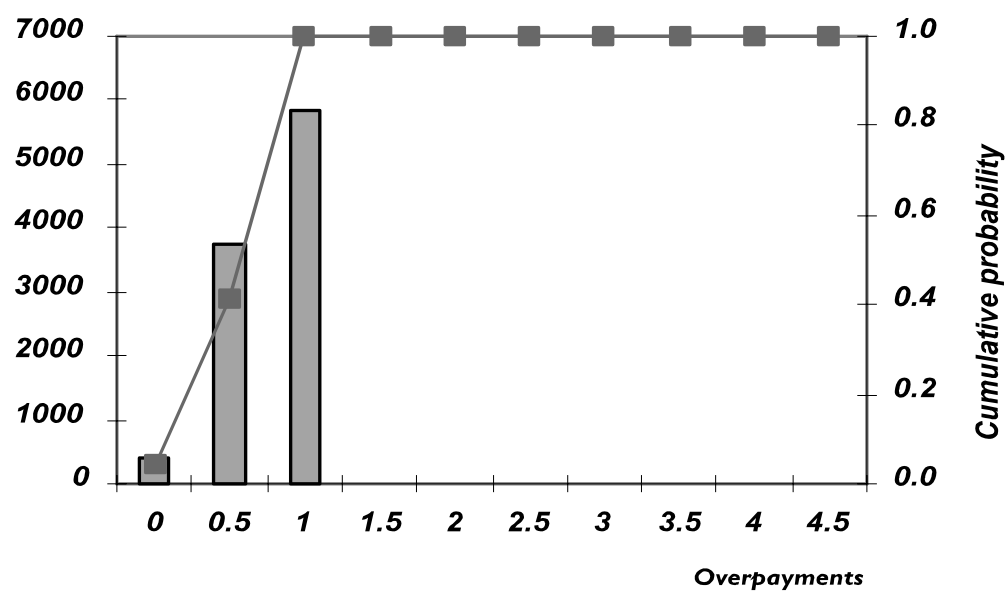

FIGURE 6.-Optimal Premiums.

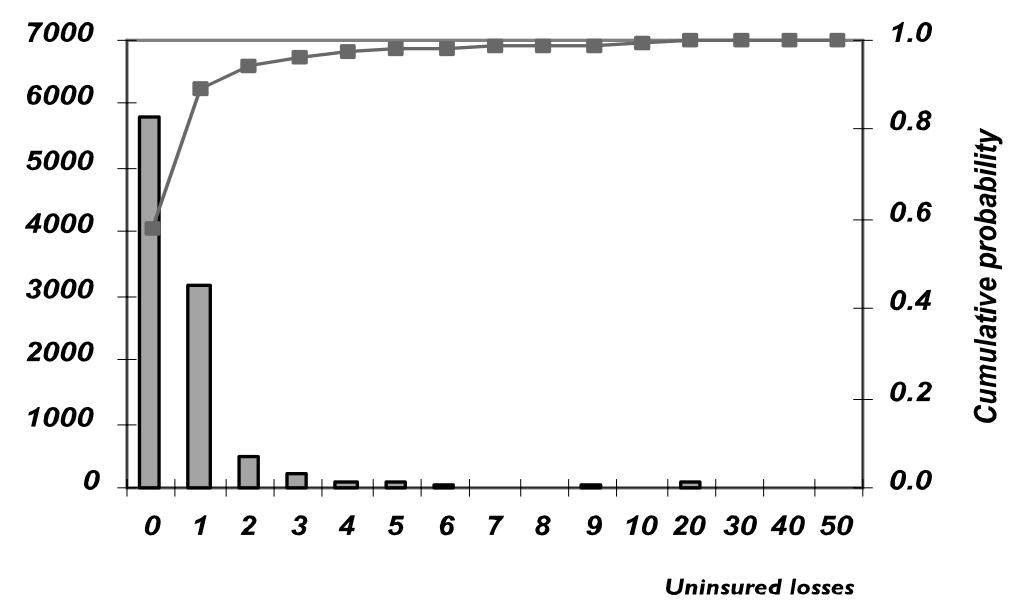

FIGURE 7.-Actuarial Premiums.

$\Sigma_{j} \max \left\{0, \tau \pi_{j}-\varphi_{j} L_{j}^{\tau}\right\} / \tau$ computed from the third term of $G(x)$.

Figures 7 and 8 show the distribution of uninsured losses computed from the first term of $G(x)$. Figures 9 and 10 show further reductions of overpayments and the demand for contingent credit for $N=20$, which are, in fact, due to increase of uncovered losses.

The computer program (optimization part) was implemented in Matlab on a DELL GX240 Personal computer. The solution time for 40 


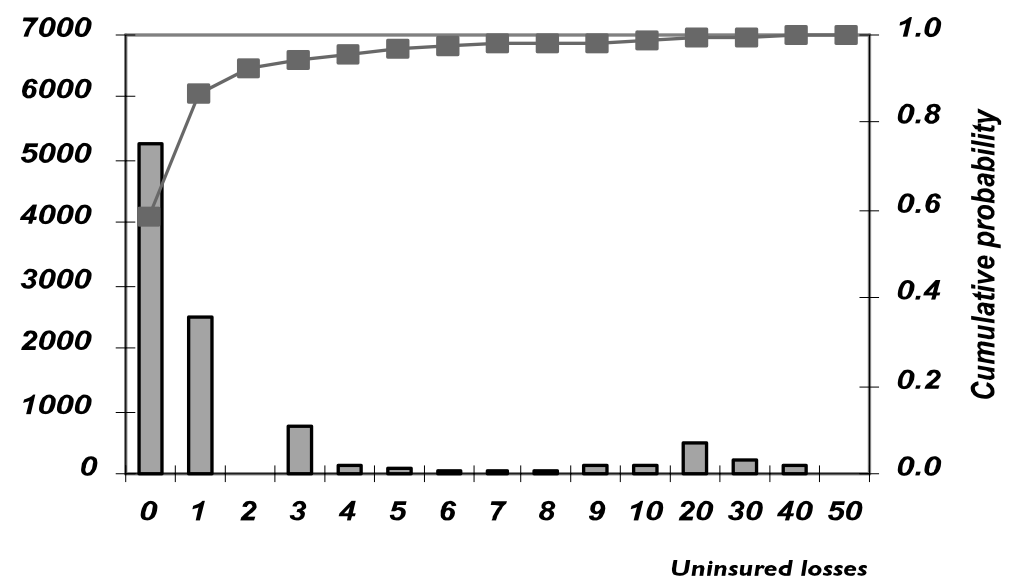

FIGURE 8.-Optimal Premiums.

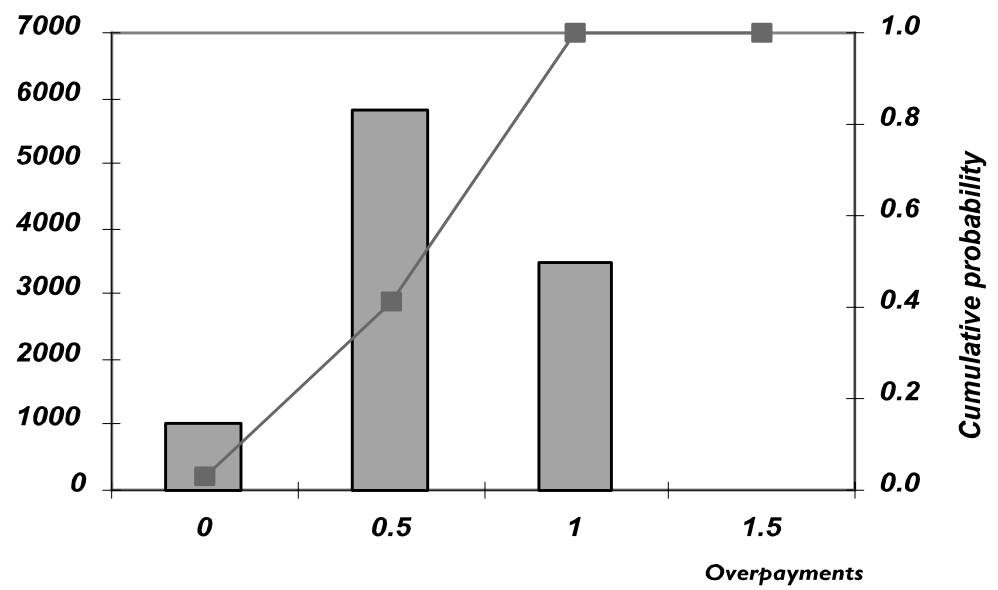

FIGURE 9.—Risk Coefficient, $N=20$.

cells and 10,000 iterations (scenarios) is 10 minutes. The optimization procedure is easily restarted from different initial solutions, for new compositions of cells, and distributions of random parameters. The solution time slightly changes with the number of decision variables and random parameters. It may increase with the increase of (unreasonably large may cause degeneracy of c- level sets) and it also depends on the rarity of catastrophes. An important idea to reduce this time is to use faster versions of the Monte Carlo simulations. 


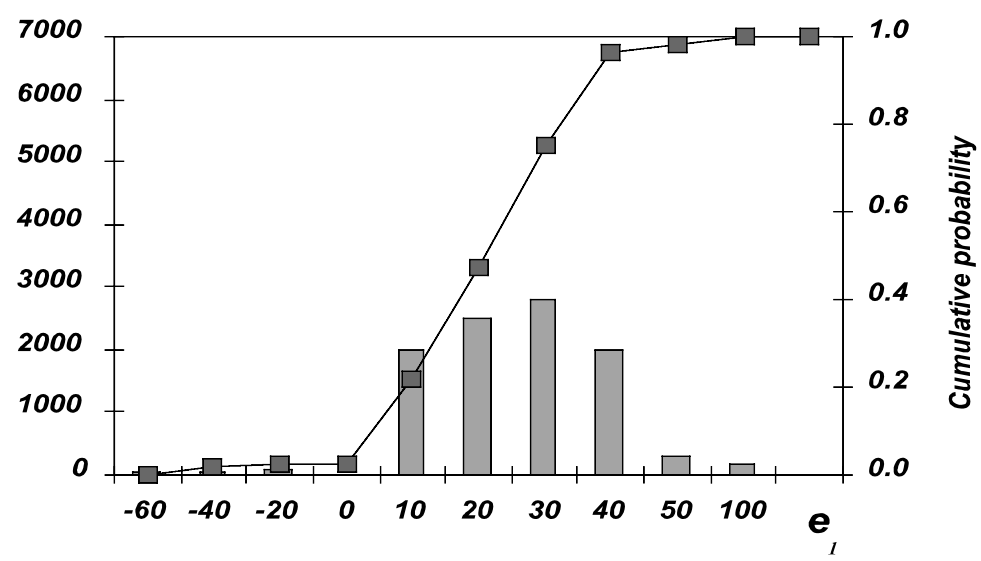

FIGURE 10.- Risk Coefficient, $N=20$.

\section{Concluding Remarks}

Traditionally, the insurance industry manages independent risks by pooling its exposures through contracts written on the basis of rich historical data. This is not possible for rare catastrophic risks with mutually dependent losses of high consequences. The proposed optimization model incorporates a generator of catastrophic losses (catastrophe model) allowing to optimize effects of dependent and location-specific rare risks through iterative improvements of the catastrophic exposures. An essential challenge is to develop a reduced version of the catastrophe model allowing fast simulation of catastrophes. It is important to understand that the main purpose of our model is not to predict catastrophes but to provide insights into robust combinations of different risk management decisions, increasing the preparedness of the region for probable catastrophes.

We demonstrated that the demand for a contingent credit significantly depends on other pillars of the loss-spreading program for the pilot region of the Upper Tisza river, Hungary. This corresponds to the conclusion of Mayers and Smith (1983) on interdependencies of individual portfolio decisions and the decision for insurance.

In particular, our numerical experiments show that optimal location-specific premiums of the mandatory public catastrophe 
insurance decrease the demand for contingent credit. It is easy to extend this analysis to derive the optimal size of contingent credit. In particular, an important task is to analyze effects of different risk reduction measures, such as strengthening or removing some of the dikes.

Purely "toy" exercises show that the diversion of capital from direct compensations to investments into loss reduction measures may essentially reduce probability of losses. As a result, it increases coverages of the mandatory public catastrophe insurance and reduces the demand for cross-subsidies on country-wide and international levels; that is, the demand for contingent credit. It is also important to analyze the effects of adaptive sampling procedures (adaptive Monte Carlo simulations), i.e., to enrich the set of decisions by components controlling the efficiency of the sampling.

The reduction of highly nonlinear and often discontinuous insolvency constraint (4) to a minimization of a non-differentiable and, in our case, a convex function (5) is a rather promising idea. The minimization of function (5) can be viewed as a version of the so-called two-stage stochastic optimization methods and stochastic min-max problems focusing our analysis on critical extreme values. The dependency of the proposed models on the stopping time brings new challenges only briefly outlined in this paper.

\section{References}

Amendola, A.; Ermoliev, Y.; Ermolieva, T.; Gitits, V.; Koff, G.; and Linnerooth-Bayer, J. 2000. A systems approach to modeling catastrophic risk and insurability. Natural Hazards Journal 21(2/3).

Amendola, A.; Ermoliev, Y.; and Ermolieva, T. 2000. Earthquake risk management: A case study for an Italian region. Proceedings of the Second EuroConference on Global Change and Catastrophe Risk Management: Earthquake Risks in Europe. Int. Inst. For Applied Systems Analysis (IIASA), Laxenburg, Austria (6-9 July).

Arrow, K. 1996. The theory of risk-bearing: small and great risks. Journal of Risk and Uncertainty 12: 103-111.

Artzner, P.; Delbaen, F.; Eber, J.-M.; and Heath, D. 1999. Coherent measures of risk. Mathematical Finance 9(3): 203-228.

Borch, K. 1962. Equilibrium in a reinsurance market. Econometrica 30(3): 424-444.

Catastrophes, Infrastructure and Poverty. 1999. Options, Int. Inst. For Applied Systems Analysis (IIASA). Laxenburg, Austria.

Climate Change and Increase in Loss Trend Persistence. 1999. Munich, 
Germany: Press Release Munich Re.

Cummins, J., D., Doherty, N. 1996. Can insurer pay for the "big one"? Measuring capacity of an insurance market to respond to catastrophic losses. Working Paper. Philadelphia: University of Pennsylvania.

Embrechts, P.; Klueppelberg, C.; and Mikosch, T. 2000. Modeling extremal events for insurance and finance. Applications of Mathematics, Stochastic Modeling and Applied Probability. Heidelberg: Springeer Verlag

Ermolieva, T.; Ermoliev, Y.; and Norkin, V. 1997. Spatial stochastic model for optimization capacity of insurance networks under dependent catastrophic risks: numerical experiments. Int. Inst. For Applied Systems Analysis (IIASA) Interim Report IR-97-028. Laxenburg, Austria.

Ermolieva, T. 1997. The design of optimal insurance decisions in the presence of catastrophic risks, Int. Inst. For Applied Systems Analysis (IIASA) Interim Report IR-97-068. Laxenburg, Austria.

Ermoliev, Y.; Ermolieva, T.; MacDonald, G.; and Norkin, V. 2000. Stochastic optimization of insurance portfolios for managing exposure to catastrophic risks. Annals of Operations Research 99: 207-225.

Ermoliev, Y.; Ermolieva, T.; MacDonald, G.; and Norkin, V. 2001. Problems on insurance of catastrophic risks. Cybernetics and Systems Analysis 37(2): 220-234.

Ermoliev, Y. and Wets, R. (eds.). 1988. Numerical Techniques of Stochastic Optimization. Computational Mathematics. Berlin: Springer Verlag.

Ermolieva, T.; Ermoliev, Y.; Hepburn, C.; Nilsson, S.; and Obersteiner M. 2003. Induced discounting and its implications to catastrophic risk management, Int. Inst. For Applied Systems Analysis (IIASA) Interim Report IR-03-029. Laxenburg, Austria.

Froot, K. 1997. The limited financing of catastrophe risk: An overview. Harvard Business School and NBER.

Jobst, N. and Zenios, S. 2001. The tail that wags the dog: integrating credit risk in asset portfolios. Journal of Risk Finance.

Kleindorfer, P. and Kunreuther, H. The complementary roles of mitigation and insurance in managing catastrophic risks. Journal of Risk Analysis, forthcoming.

Kunreuther, H. and Linnerooth-Bayer, J. 2000. The financial management of catastrophic flood risks in emerging economy countries. Proceedings of the Second EuroConference on Global Change and Catastrophe Risk Management: Earthquake Risks in Europe. Int. Inst. For Applied Systems Analysis (IIASA), Laxenburg, Austria (6-9 July).

Linnerooth-Bayer, J. and Amendola, A. 2000. Global change, catastrophic risk and loss spreading. The Geneva Papers on Risk and Insurance 25(2): 203-219.

Mayers, D. and Smith, C. 1983. The interdependencies of individual portfolio decisions and the demand for insurance. Journal of Political Economy 91(2): 304-311. 
National Research Council. 1999. National disaster losses: a framework for assessment. Committee on assessing the costs of natural disasters, Washington D.C.: National Academy Press.

Pollner, J. 2000. Catastrophe risk management: using alternative risk financing and insurance pooling mechanisms, in Finance, Private Sector \& Infrastructure Sector Unit, Caribbean Country Department, Latin America and the Caribbean Region. World Bank.

Proposal for the Project on Flood Risk Management Policy in the Upper Tisza Basin: A System Analytical Approach. Int. Inst. For Applied Systems Analysis (IIASA), 2000.

Pugh, E.L. 1966. A gradient technique of adaptive Monte Carlo. SIAM Review 8(3): 346-355.

Rockafellar, T. and Uryasev, S. 2000. Optimization of conditional value-at-risk. The Journal of Risk 2: 21-41.

Rundle, Turcotte, Klein (eds.). 1996. Reduction and Protection of Natural Disasters. Addison-Wesley.

Stone, J.M. 1973. A theory of capacity and the insurance of catastrophe risks, parts 1, 2. The Journal of Risk and Insurance 40: 231-244 and 339-355.

Thomas, F. 1994. Principles of flood plain management. Proceedings of the NATO Advanced Study Institute on Defense from Floods and Floodplain Management. Dordrechtt, The Netherlands: Kluwer Academic Publishers.

Walker, G. 1997. Current developments in catastrophe modelling. In: N.R. Britton and J. Oliver (eds.). Financial Risks Management for Natural Catastrophes. Brisbane, Griffith University, Australia: 17-35.

Yang, H. 2000. An integrated risk management method: VaR approach. Multinational Finance Journal 4: 201-219. 